\title{
NOTES ON THE THEORY AND APPLICATION OF FOURIER TRANSFORMS. III, IV, V, VI, VII*
}

BY

R. E. A. C. PALEY AND N. WIENER

III. ON MÜNTZ'S THEOREM

1. We give a proof of the following theorem which is Szász'sł generalization of Müntz's $\ddagger$ theorem. The method is similar to one employed by Carleman§ to prove Müntz's theorem. Incidentally we give a theorem concerning the distribution of the zeros of functions analytic in a half-plane, which is analogous to, and in some respects more general than, another theorem of Carleman's paper.

2. We recall the following well known

TheOREM I. Let

$$
\zeta_{n}=\rho_{n} e^{i \theta_{n}}, 0 \leqq \rho_{n}<1 \quad(n=1,2, \cdots)
$$

be a set of points in the unit circle $|\zeta|<1$. Suppose that an analytic function $f_{1}(\zeta)$ is regular in $|\zeta|<1$, satisfies

$$
\int_{-\pi}^{\pi}\left|f_{1}\left(\rho e^{i \theta}\right)\right|^{2} d \theta \leqq B, \rho<1,
$$

where $B$ is a constant which depends only on $f_{1}$, and has zeros at the points $\zeta_{n}$. Then

$$
\sum_{n=1}^{\infty}\left(1-\rho_{n}\right)<\infty .
$$

Conversely, if the series (2.3) converges, then there exists a bounded function $f_{1}(\zeta)$ which has zeros at the points $\zeta_{n}$.

Suppose we invert the interior of the unit circle into the half-plane $\Im(z)>0$, by means of the substitution

* Presented to the Society, October 28, 1933; received by the editors March 16, 1933. Notes I and II of the Series have appeared in this volume of these Transactions, pp. 348-355.

† O. Szász, Über die Approximation stetiger Funktionen durch lineare Aggregate von Potensen, Mathematische Annalen, vol. 77 (1916), pp. 482-496.

† C. H. Müntz, Über den Approximationssatz von Weierstrass, Schwarz's Festschrift, Berlin, 1914, pp. 303-312.

8 T. Carleman, Über die Approximation analytischer Funktionen durch lineare Aggregate son vorgegebenen Potenzen, Arkiv för Matematik, Astronomi och Fysik, vol. 17 (1922-23), No. 9, pp. 1-30. 


$$
\zeta=\frac{1+i z}{1-i z}
$$

Suppose that the point $\zeta=\rho e^{i \theta}$ inverts into $z=x+i y$. Then it is a matter of elementary algebra to verify that the convergence of the series (2.3) is equivat lent to that of the series

$$
\sum_{n=1}^{\infty} \frac{y_{n}}{1+x_{n}^{2}+y_{n}^{2}}
$$

$z_{n}=x_{n}+i y_{n}, y_{n}>0$, being the inverse of $\zeta_{n}=\rho_{n} e^{i \theta_{n}}$.

We are now in a position to prove our next theorem, which is a generalization of Carleman's:

THEOREM II. Let

$$
z_{n}=x_{n}+i y_{n} \quad(n=1,2, \cdots)
$$

denote a sequence of points in the half plane $\Im(z)>0$, and let $f(z)$ be a function regular in $\Im(z)>0$ which satisfies

$$
\int_{-\infty}^{\infty}|f(x+i y)|^{2} d x<1
$$

and has zeros at the points $z_{n}$. Then

$$
\sum_{n=1}^{\infty} \frac{y_{n}}{1+x_{n}^{2}+y_{n}^{2}}<\infty .
$$

Conversely, if the series (2.7) converges, then there exists a bounded function $f(z)$ regular in $\Im(z)>0$, satisfying (2.6) and having zeros at the points $z_{n}$.

For the second half of the theorem we have only to observe, by Theorem I, that there exists a function $g(z)$ with zeros at the points $z_{n}$, analytic in $\Im(z)>0$, and less than 1 in absolute value. We have now only to write

$$
f(z)=\frac{1}{2}(z+i)^{-1} g(z)
$$

and condition (2.6) is satisfied.

To prove the first part of the Theorem it will be sufficient to show that the function $f(z)$ can be represented by a Cauchy integral

$$
f(z)=(2 \pi i)^{-1} \int_{-\infty}^{\infty} \frac{f^{*}\left(x^{\prime}\right)}{x^{\prime}-z} d x^{\prime}, \Im(z)>0,
$$

where the function $f^{*}\left(x^{\prime}\right)$ satisfies

$$
\int_{-\infty}^{\infty}\left|f^{*}\left(x^{\prime}\right)\right|^{2} d x^{\prime} \leqq 1
$$


Indeed, it is readily seen that the substitution (2.4) transforms the integral of the right-hand member of (2.8) into

$$
(2 \pi i)^{-1} \int_{-\pi}^{\pi} \frac{f_{1}^{*}\left(\zeta^{\prime}\right) d \zeta^{\prime}}{\zeta^{\prime}-\zeta}+C, \zeta^{\prime}=e^{i \phi},
$$

where $f_{1}^{*}\left(\zeta^{\prime}\right)$ corresponds to $f^{*}\left(x^{\prime}\right)$ and

$$
C=(2 \pi i)^{-1} \int_{-\infty}^{\infty} \frac{f^{*}\left(x^{\prime}\right)}{x^{\prime}+i} d x^{\prime} .
$$

It follows that, on putting $f_{1}(\zeta) \equiv f(z)$,

$$
\int_{-\pi}^{\pi}\left|f_{1}\left(\rho e^{i \theta}\right)\right|^{2} d t \leqq B
$$

and we may apply Theorem I.

Now suppose that

$$
0<\epsilon<y<y_{0}, \quad 2|x|<N_{0}<N .
$$

Then

$$
\begin{aligned}
2 \pi i f(z)= & \int_{-N}^{N} \frac{f\left(x^{\prime}+i \epsilon\right)}{x^{\prime}+i \epsilon-z} d x^{\prime}-\int_{-N}^{N} \frac{f\left(x^{\prime}+i y_{0}\right)}{x^{\prime}+i y-z} d x^{\prime} \\
& +i \int_{0}^{y_{0}} \frac{f\left(N+i y^{\prime}\right)}{N+i y^{\prime}-z} d y^{\prime}-i \int_{0}^{y_{0}} \frac{f\left(-N+i y^{\prime}\right)}{-N+i y^{\prime}-z} d y^{\prime} .
\end{aligned}
$$

But

$$
\begin{aligned}
\frac{1}{N_{0}} \int_{N_{0}}^{2 N_{0}} d N & \int_{.}^{\nu_{0}} \frac{\left|f\left(N+i y^{\prime}\right)\right|}{\left|N+i y^{\prime}-z\right|} d y^{\prime} \leqq \frac{2}{N_{0}^{2}} \int_{0}^{\nu_{0}} d y^{\prime} \int_{N_{0}}^{2 N_{0}}\left|f\left(N+i y^{\prime}\right)\right| d N \\
& \leqq \frac{2}{N_{0}^{2}} \int_{0}^{y_{0}} d y^{\prime} N_{0}^{1 / 2}\left[\int_{N_{0}}^{2 N_{0}}\left|f\left(N+i y^{\prime}\right)\right|^{2} d N\right]^{1 / 2} \leqq 2\left(y_{0}-\epsilon\right) N_{0}-3 / 2
\end{aligned}
$$

and tends to zero as $N_{0} \rightarrow \infty$. Similar analysis applies to the last term. Hence

$$
\begin{aligned}
2 \pi i f(z) & =\lim _{N_{0} \rightarrow \infty} \frac{1}{N_{0}} \int_{N_{0}}^{2 N_{0}} d N\left[\int_{-N}^{N} \frac{f\left(x^{\prime}+i \epsilon\right)}{x^{\prime}+i \epsilon-z} d x^{\prime}-\int_{-N}^{N} \frac{f\left(x^{\prime}+i y_{0}\right)}{x^{\prime}+i y_{0}-z} d x^{\prime}\right] \\
& =\int_{-\infty}^{\infty} \frac{f\left(x^{\prime}+i \epsilon\right)}{x^{\prime}+i \epsilon-z} d x^{\prime}-\int_{-\infty}^{\infty} \frac{f\left(x^{\prime}+i y_{0}\right)}{x^{\prime}+i y_{0}-z} d x^{\prime},
\end{aligned}
$$

since the last two integrals converge absolutely. Now make $y_{0}$ tend to infinity. The second integral does not exceed 


$$
\left[\int_{-\infty}^{\infty}\left|f\left(x^{\prime}+i y_{0}\right)\right|^{2} d x^{\prime}\right]^{1 / 2}\left[\int_{-\infty}^{\infty} \frac{d x^{\prime}}{\left|x^{\prime}+i y_{0}-z\right|^{2}}\right]^{1 / 2},
$$

and so tends to zero.

Since condition (2.6) is satisfied, by a classical argument of F. Riesz $\dagger$ there exists a sequence $\left\{\epsilon_{k} \downarrow 0\right\}$ such that $f\left(x+i \epsilon_{k}\right)$ converges weakly to a function $f^{*}(x)$ satisfying (2.9). Hence

$$
2 \pi i f(z)=\int_{-\infty}^{\infty} \frac{f\left(x^{\prime}+i \epsilon_{k}\right)}{x^{\prime}-z} d x^{\prime} \rightarrow \int_{-\infty}^{\infty} \frac{f^{*}\left(x^{\prime}\right)}{x^{\prime}-z} d x^{\prime} \text { as } \epsilon_{k} \rightarrow 0,
$$

which is the desired result.

3. We proceed now to our main theorem.

ThEOREM III. A necessary and sufficient condition for the closure $L^{2}$ of the functions $t_{n}, \Re\left(\lambda_{n}\right)>-\frac{1}{2}$, in the interval $(0,1)$, is the divergence of the series

$$
\sum_{n=1}^{\infty} \frac{1+2 \Re\left(\lambda_{n}\right)}{1+\left|\lambda_{n}\right|^{2}}
$$

We observe first that if the functions $t^{\lambda_{n}}$ are not closed $L^{2}$ then there exists a function $\phi(t)$ of integrable square on $(0,1)$ which is orthogonal to them all, so that we have

$$
\begin{aligned}
0< & \int_{0}^{1}|\phi(t)|^{2} d t<\infty, \\
& \int_{0}^{1} \phi(t) \bar{t}^{\bar{\lambda}_{n}} d t=0 \quad(n=1,2,3, \cdots) .
\end{aligned}
$$

Conversely if the system $\left\{t^{\lambda_{n}}\right\}$ is closed then there exists no function $\phi(t)$ not identically zero satisfying (3.2) and (3.3). Now let us write $t=e^{x}$; the conditions (3.2) and (3.3) become

$$
\begin{aligned}
& 0<\int_{-\infty}^{0}\left|\phi\left(e^{x}\right)\right|^{2} e^{x} d x<\infty \\
& \int_{-\infty}^{0} \phi\left(e^{x}\right) \exp \left[x\left(1+\bar{\lambda}_{n}\right)\right] d x=0 \quad(n=1,2, \cdots) .
\end{aligned}
$$

Upon writing $\phi\left(e^{x}\right) e^{x / 2}=\Phi(x)$, we transform the last two formulas into

† Untersuchungen über Systeme integrierbarer Funktionen, Mathematische Annalen, vol. 69 (1910), pp. 449-497; pp. 466-468. 


$$
\begin{aligned}
& 0<\int_{-\infty}^{0}|\Phi(x)|^{2} d x<\infty \\
& \int_{-\infty}^{0} \Phi(x) \exp \left[x\left(\frac{1}{2}+\bar{\lambda}_{n}\right)\right] d x=0 \quad(n=1,2, \cdots) .
\end{aligned}
$$

Thus the closure or non-closure $L^{2}$ of the functions $t^{\lambda_{n}}$ on $(0,1)$ is equivalent to the non-existence or existence of a function satisfying (3.4) and (3.5), which is equivalent to the closure or non-closure of the functions $\exp \left[x\left(\frac{1}{2}+\lambda_{n}\right)\right]$ on the interval $(-\infty, 0)$.

4. Proceeding now to the proof of the theorem we observe first that, if there exists a function satisfying (3.4) and (3.5), then the function

$$
f(z)=\int_{-\infty}^{0} \Phi\left(x^{\prime}\right) e^{-i z x^{\prime}} d x
$$

exists for $\Im(z)>0$, and defines an analytic function in that half-plane. Further, by $(3.5)$, it vanishes at the points $\left(\frac{1}{2}+\bar{\lambda}_{n}\right) i$. Since, by Plancherel's theorem,

$$
\int_{-\infty}^{\infty}|f(x+i y)|^{2} d x=2 \pi \int_{-\infty}^{0}\left|\Phi\left(x^{\prime}\right)\right|^{2} e^{2 y x^{\prime}} d x^{\prime}
$$

the series (3.1) converges by Theorem II. Thus the non-closure of the functions $\left\{x^{\lambda_{n}}\right\}$ implies the convergence of (3.1).

To obtain the converse we have to show that when (3.1) converges then we can find a function $\Phi(x)$ satisfying (3.4) and (3.5). Now in virtue of Theorem II we can find a function $f(z)$ which is analytic for $\Im(z)>0$, is uniformly bounded in this half-plane, and vanishes at the points $\left(\frac{1}{2}+\bar{\lambda}_{n}\right) i$, with the integral

$$
\int_{-\infty}^{\infty}|f(x+i y)|^{2} d x
$$

uniformly bounded. Let $g_{y}(\xi)$ denote the Fourier transform

$$
(2 \pi)^{-1 / 2} \int_{-\infty}^{\infty} f(x+i y) e^{i x \xi} d x
$$

of $f(x+i y)$. Then the argument given in detail in the first note of this series (Theorem II) shows that $g_{y}(\xi)$ is of the form $G(\xi) e^{y \xi}$ for $\xi<0$ and vanishes for $\xi>0$, where

$$
\int_{-\infty}^{0}|G(\xi)|^{2} d \xi<\infty
$$

Now 


$$
\begin{aligned}
(2 \pi)^{-1 / 2} & \int_{-\infty}^{0} G(\xi) \exp \left[\xi\left(\frac{1}{2}+\bar{\lambda}_{n}\right)\right] d \xi \\
& =(2 \pi)^{-1 / 2} \int_{-\infty}^{0} G(\xi) \exp \left[\xi \Re\left(\frac{1}{2}+\lambda_{n}\right)\right] \exp \left[-i \xi \Im\left(\lambda_{n}\right)\right] d \xi \\
& =(2 \pi)^{-1 / 2} \int_{-\infty}^{0} g_{y}(\xi) \exp \left[-i \xi \Im\left(\lambda_{n}\right)\right] d \xi \quad \quad\left(y=\Re\left(\frac{1}{2}+\lambda_{n}\right)\right) \\
& =f\left\{\Im\left(\lambda_{n}\right)+i \Re\left(\frac{1}{2}+\lambda_{n}\right)\right\}=f\left\{\left(\frac{1}{2}+\bar{\lambda}_{n}\right) i\right\}=0 .
\end{aligned}
$$

Thus, for all $n$,

$$
\int_{-\infty}^{0} G(\xi) \exp \left[\xi\left(\frac{1}{2}+\bar{\lambda}_{n}\right)\right] d \xi=0 .
$$

We have only to identify $\Phi(x)$ with $G(x)$ and our theorem is proved.

5. The problem of the closure in $L^{2}$ of functions $e^{\lambda_{n} x}$ on a finite interval is much more difficult than the corresponding one for an interval which is infinite in one direction. We have obtained a number of theorems in this direction but nothing like a complete answer to the problem. In the case however where all the numbers $\lambda_{n}$ are real (we need no longer assume that $\lambda_{n}$ is positive or negative) a necessary and sufficient condition for the closure of the functions $e^{\lambda_{n} x}$ on a finite interval is the divergence of the series

$$
\sum_{n=1}^{\infty} \frac{1}{1+\left|\lambda_{n}\right|}
$$

\section{A THEOREM ON CLOSURE}

1. The present note is devoted to the proof of the following theorem:

ThEOREM I. The set of functions $\left\{e^{-\pi|x| / 2} e^{i \lambda_{n} x}\right\}$ is closed $L^{2}$ over $(-\infty, \infty)$ when and only when

$$
\sum_{n=1}^{\infty} \frac{\cos \Im\left(\lambda_{n}\right)}{\cosh \Re\left(\lambda_{n}\right)}=\infty,-\frac{1}{2} \pi<\Im\left(\lambda_{n}\right)<\frac{1}{2} \pi .
$$

The condition (1.1) should be contrasted with the condition

$$
\sum_{n=1}^{\infty} \frac{\Im\left(\lambda_{n}\right)-\frac{1}{2} \pi}{1+\left|\lambda_{n}\right|^{2}}=\infty, \Im\left(\lambda_{n}\right)>-\frac{1}{2} \pi,
$$

which is a necessary and sufficient condition for the closure $L^{2}$ of the funcs tions $\left\{e^{-\pi|x| / 2} e^{\lambda_{n} x}\right\}$ on the interval $(0, \infty)$ (see e.g. the preceding note of this series). Thus if for instance all the numbers $\lambda_{n}$ are real, the conditions for closure on the intervals $(0, \infty)$ and $(-\infty, \infty)$ are 


$$
\sum_{n=1}^{\infty} \frac{1}{1+\left|\lambda_{n}\right|^{2}}=\infty, \sum_{n=1}^{\infty} e^{-\left|\lambda_{n}\right|}=\infty
$$

respectively.

2. We shall prove the theorem by the following chain of lemmas:

LEMaA 1. As $|x| \rightarrow \infty$ in either direction along the real axis,

$$
\left|\Gamma\left(i x+\frac{1}{2}\right)\right| \sim(2 \pi)^{1 / 2} e^{-\pi|x| / 2} .
$$

This is an immediate consequence of Stirling's formula.

LEMac 2. The set of functions $\left\{e^{-x|x| / 2} e^{i_{n} x}\right\}$ is closed $L^{2}$ when and only when the set $\left\{\Gamma\left(i x+\frac{1}{2}\right) e^{i \lambda_{n} x}\right\}$ is closed $L^{2}$.

For let

$$
\int_{-\infty}^{\infty}|f(x)|^{2} d x<\infty
$$

and

$$
\int_{-\infty}^{\infty} f(x) \Gamma\left(i x+\frac{1}{2}\right) e^{i \lambda_{n} x} d x=0 \quad(n=1,2, \cdots)
$$

Let

$$
g(x)=f(x) \Gamma\left(i x+\frac{1}{2}\right) e^{\pi|x| / 2} \text {. }
$$

Then, by Lemma 1,

$$
\int_{-\infty}^{\infty}|g(x)|^{2} d x<\infty
$$

and

$$
\int_{-\infty}^{\infty} g(x) e^{-\pi|x| / 2} e^{i \lambda_{n} x} d x=0 \quad(n=1,2, \cdots)
$$

Similarly, (2.1) and (2.2) follow from (2.3) and (2.4). Thus there exists a function $f(x)$ orthogonal to the set $\left\{\Gamma\left(i x+\frac{1}{2}\right) e^{i \lambda_{n} x}\right\}$ when and only when there exists a function $g(x)$ orthogonal to the set $\left\{e^{-\pi|x| / 2} e^{a_{n} x}\right\}$, and one set is closed $L^{2}$ when and only when the other set is closed.

LEMMA 3. The set of functions

$$
e^{v / 2} \exp \left(-e^{v-\lambda_{n}}\right) \quad(n=1,2, \cdots)
$$

is closed $L^{2}$ over $(-\infty, \infty)$ when and only when the set of functions $\left\{\Gamma\left(i x+\frac{1}{2}\right) e^{i \lambda_{n} x}\right\}$ is closed $L^{2}$ over $(-\infty, \infty)$. 
This follows from the fact that the Fourier transform of (2.5), apart from a constant factor, is $\Gamma\left(i x+\frac{1}{2}\right) e^{i \lambda_{n} x}$, and that, by Plancherel's theorem, $L^{2}$-closure is invariant under a Fourier transformation.

LEMara 4. The set of functions $\left\{x^{\mu_{n}}\right\}, \mu_{n}=e^{-\lambda_{n}}-\frac{1}{2}$, is closed $L^{2}$ over $(0,1)$ when and only when the set of functions $(2.5)$ is closed $L^{2}$ over $(-\infty, \infty)$.

For the pair of statements

$$
\int_{0}^{1}|f(x)|^{2} d x<\infty
$$

and

$$
\int_{0}^{1} f(x) x^{\operatorname{mn}} d x=0
$$

is equivalent to the pair of statements

$$
\int_{-\infty}^{\infty}|g(v)|^{2} d v=0
$$

and

$$
\int_{-\infty}^{\infty} g(v) e^{v / 2} \exp \left(-e^{v-\lambda_{n}}\right) d v=0,
$$

where

$$
g(v)=f\left[\exp \left(-e^{v}\right)\right] e^{v / 2} \exp \left(-e^{v} / 2\right),
$$

and we may again apply the argument of Lemma 2.

Lemar 5. The set of functions $\left\{x^{\omega_{n}}\right\}, \Re\left(\mu_{n}\right)>-\frac{1}{2}$, is closed $L^{2}$ over $(0,1)$ when and only when

$$
\sum_{n=1}^{\infty} \frac{2 \Re\left(\mu_{n}\right)+1}{1+\left|\mu_{n}\right|^{2}}=\infty
$$

This is a well known theorem of Szász. $\dagger$

Condition (2.6) is equivalent to (1.1). Theorem I now follows by combining Lemmas 2, 3, 4, and 5 .

\section{ON ENTIRE FUNCTIONS}

1. Let $f(z)$ be an entire function, $f(0)=1$, and $\left\{z_{v}\right\}$ the sequence of zeros of $f(z)$. We denote by $M_{f}(r)$ and $m_{f}(r)$ respectively the maximum and minimum of $|f(z)|$ on the circle $|z|=r$, and by $n_{f}(r)$ the number of zeros of $f(z)$

† Loc. cit. See also our preceding note. 
contained in the interior of this circle. The purpose of this note is to prove the following two theorems:

ThEOREM I. Let

$$
\log M_{f}(r)=O\left(r^{1 / 2}\right)
$$

and

$$
\int_{0}^{\infty} \log +m_{f}(r) r^{-3 / 2} d r<\infty
$$

Then

$$
n_{f}(r) \sim A r^{1 / 2}
$$

where the constant $A$ is determined by

$$
A=-\pi^{-2} \int_{0}^{\infty} \log \prod_{v=1}^{\infty}\left|\left(1-\frac{x}{\left|z_{v}\right|}\right)\right| x^{-3 / 2} d x .
$$

THEOREM II. Let $f(z)$ be an entire function of order not exceeding $\frac{1}{2}$. If the conditions

$$
\begin{gathered}
n_{f}(r) \sim B r^{1 / 2}, \\
B=-\pi^{-2} \int_{0}^{\infty} \log |f(x)| x^{-3 / 2} d x, \quad f(0)=1,
\end{gathered}
$$

are satisfied, then all roots of $f(z)$ are positive.

The proofs of these theorems are based upon a lemma which is of independent interest. This lemma is discussed in the next $\$ 2$. In $\$ 3$ we give proofs of Theorems I and II. In $\$ 4$ we give a modification of the lemma of $\$ 2$ and discuss its application to the theory of the Riemann zeta-function. In the last $\$ 5$ we give proof of some results analogous to those of $\$ 2$. They are in part contained in a paper by Titchmarsh, $\dagger$ and in part represent extensions of his results.

2. Let $\left\{\lambda_{\nu}\right\}$ be a monotone sequence of positive numbers such that the series $\sum_{1}^{\infty} \lambda_{\nu}^{-2}$ converges. We set

$$
\phi(z)=\prod_{v=1}^{\infty}\left(1-\frac{z^{2}}{\lambda_{v}^{2}}\right) .
$$

†E. C. Titchmarsh, On integral functions with real negative zeros, Proceedings of the London Mathematical Society, (2), vol. 26 (1927), pp. 185-200. 
LEMMa 2.1. If $\sum_{1}^{\infty} \lambda_{i}^{-2}$ converges then the statements

$$
\log \phi(i y) \sim \pi A|y| \text { as }|y| \rightarrow \infty,
$$

$$
\int_{-\infty}^{\infty} \log |\phi(x)| x^{-2} d x=-\pi^{2} A,
$$

are completely equivalent.

We have, assuming $y>0$,

$$
\begin{aligned}
(\pi y)^{-1} \log \phi(i y) & =(\pi y)^{-1} \sum_{p=1}^{\infty} \log \left(1+\frac{y^{2}}{\lambda_{p}^{2}}\right) \\
& =(\pi y)^{-1} \int_{0}^{\infty} \log \left(1+\frac{y^{2}}{t^{2}}\right) d \Lambda(t)
\end{aligned}
$$

where $\Lambda(t)$ is the number of $\lambda_{\nu}$ 's not exceeding $t$. Similarly

$$
\begin{aligned}
- & \pi^{-2} \int_{-y}^{y} \log |\phi(x)| x^{-2} d x=-2 \pi^{-2} \int_{0}^{\nu} x^{-2} d x \int_{0}^{\infty} \log \left|1-\frac{x^{2}}{t^{2}}\right| d \Lambda(t) \\
& =-2 \pi^{-2} \int_{0}^{\infty} d \Lambda(t) \int_{0}^{y} \log \left|1-\frac{x^{2}}{t^{2}}\right|_{0}^{x^{-2} d x} \\
& =-2 \pi^{-2} y^{-1} \int_{0}^{\infty} d \Lambda(t) \frac{y}{t} \int_{0}^{y / t} \log \left|1-s^{2}\right| s^{-2} d s .
\end{aligned}
$$

Expressions (2.4), (2.5) are both of the form

$$
\frac{1}{y} \int_{0}^{\infty} N\left(\frac{t}{y}\right) d \Lambda(t)
$$

where $\Lambda(t)$ is a monotone increasing function. In (2.4) we have

$$
N(\lambda)=N_{1}(\lambda)=\frac{1}{\pi} \log \left(1+\frac{1}{\lambda^{2}}\right),
$$

while, in (2.5),

$$
\begin{aligned}
N(\lambda) & =N_{2}(\lambda)=-\frac{2}{\pi^{2} \lambda} \int_{0}^{1 / \lambda} \log \left|1-x^{2}\right| x^{-2} d x \\
& =\frac{2}{\pi^{2}}\left\{\log \left|1-\frac{1}{\lambda^{2}}\right|+\frac{1}{\lambda} \log \left|\frac{1+\lambda}{1-\lambda}\right|\right\} .
\end{aligned}
$$

The function $N_{1}(\lambda)$ is positive and monotone decreasing, the same being also true of $N_{2}(\lambda)$ since 


$$
N_{2}^{\prime}(\lambda)=-\frac{2}{\pi^{2} \lambda^{2}} \log \left|\frac{1+\lambda}{1-\lambda}\right| \text {. }
$$

If we write $N(\lambda)$ for either of $N_{1}(\lambda), N_{2}(\lambda)$, the following properties are easily established:

$$
N(\lambda)=\left\{\begin{array}{l}
o\left(\log \frac{1}{\lambda}\right) \text { as } \lambda \rightarrow 0, \\
o\left(\frac{1}{\lambda^{2}}\right) \text { as } \lambda \rightarrow \infty
\end{array}\right.
$$

$$
\sum_{k=-\infty}^{\infty} \max _{2^{k} \leq \lambda \leq 2^{k+1}} \lambda N(\lambda)<\infty, N(\lambda)>0
$$

$$
\begin{aligned}
& \int_{0}^{\infty} N_{1}(\lambda) \lambda^{i t} d \lambda=\frac{1}{\pi(i t+1)} \int_{0}^{\infty} \frac{\mu^{(i t-1) / 2} d \mu}{1+\mu}=\frac{1}{(i t+1) \cosh \frac{\pi t}{2}}, \\
& \int_{0}^{\infty} N_{2}(\lambda) \lambda^{i t} d \lambda=\frac{2}{\pi^{2}(i t+1)} \int_{0}^{\infty} \lambda^{i t-1} \log \left|\frac{1+\lambda}{1-\lambda}\right| d \lambda
\end{aligned}
$$

$$
=\frac{2}{\pi^{2}(i t+1)} \sum_{k=-\infty}^{\infty} \frac{1}{\left(\frac{t}{2}\right)^{2}+\left(k+\frac{1}{2}\right)^{2}}=\frac{2 \tan \frac{\pi i t}{2}}{\pi i t(i t+1)} .
$$

It follows that

$$
\begin{aligned}
& \int_{0}^{\infty} N(\lambda) \lambda^{i t} d \lambda \neq 0 \text { when } t \text { is real, } \\
& \int_{0}^{\infty} N(\lambda) d \lambda=1 .
\end{aligned}
$$

We observe finally that the expressions

$$
\begin{aligned}
& \frac{1}{y} \int_{0}^{\infty} N_{1}\left(\frac{t}{y}\right) d \Lambda(t)=(\pi y)^{-1} \log \phi(i y), \\
& \frac{1}{y} \int_{0}^{\infty} N_{2}\left(\frac{t}{y}\right) d \Lambda(t)=-\pi^{-2} \int_{-y}^{y} \log |\phi(x)| x^{-2} d x
\end{aligned}
$$

$\rightarrow 0$ as $y \rightarrow 0$. Hence either of the statements

$$
\frac{1}{y} \int_{0}^{\infty} N_{i}\left(\frac{t}{y}\right) d \Lambda(t) \rightarrow A \text { as } y \rightarrow \infty, i=1,2,
$$


implies the boundedness of the corresponding integral

$$
\frac{1}{y} \int_{0}^{\infty} N_{i}\left(\frac{t}{y}\right) d \Lambda(t)
$$

over the range $(0, \infty)$. A direct application of a Tauberian theorem of Wiener $\dagger$ shows that statements (2.14) are completely equivalent, which is precisely the result of our Lemma 2.1.

3. We now proceed to the proofs of Theorems 1 and 2.

Proof of Theorem I. We first observe that by a known theorem the assertions $\log M_{f}(r)=O\left(r^{1 / 2}\right)$ and $n_{f}(r)=O\left(r^{1 / 2}\right)$ are equivalent. It follows that if we replace each zero by another zero with the same absolute value but situated on the positive part of the real axis, changing in effect

$$
\prod_{1}^{\infty}\left(1-\frac{z}{z_{v}}\right) \text { into } \prod_{1}^{\infty}\left(1-\frac{z}{\left|z_{v}\right|}\right),
$$

we certainly do not affect the truth of (1.1). Secondly this process will decrease $m_{f}(r)$ for every value of $r$, so that we do not affect the truth of (1.2) either. Thus it is legitimate to assume that all the zeros of $f(z)$ are real and positive. Let them be

$$
\lambda_{1}{ }^{2}, \lambda_{2}{ }^{2}, \cdots ; \quad 0<\lambda_{1} \leqq \lambda_{2} \leqq \cdots ; \sum_{1}^{\infty} \lambda_{\nu}{ }^{2}<\infty .
$$

Our next observation is that, by some theorems of Titchmarsh $\ddagger$, for a function of this special type the assertions

$$
n_{f}(r) \sim A r^{1 / 2} \text { and } \log M_{f}(r) \sim \pi A r^{1 / 2}
$$

are equivalent, so that we may leave $n_{f}(r)$ and confine our attention to $M_{f}(r)$. We write

$$
z^{1 / 2}=w=u+i v
$$

so that $f(z)$ is transformed into the new function

$$
f\left(w^{2}\right)=(w)=\prod_{n=1}^{\infty}\left(1-\frac{w^{2}}{\lambda_{\nu}^{2}}\right)
$$

which satisfies

$$
\log ^{+}|\phi(w)|=O(|w|)
$$

and

$\dagger$ N. Wiener, Tauberian theorems, Annals of Mathematics, (2), vol. 33 (1932), pp. 1-100; Theorem $\mathrm{XI}^{\prime}$, p. 30 .

$\ddagger$ Loc. cit., Theorems I and II. 


$$
\int_{-\infty}^{\infty} \log ^{+}|\phi(u)| u^{-2} d u<\infty \text {. }
$$

We have to show that

$$
\log \phi(i v) \sim \pi A|v|
$$

Since the series $\sum_{1}^{\infty} \lambda_{\nu}^{-2}$ converges, by Lemma 2.1 it will be sufficient to establish the convergence of the integral

$$
\int_{-\infty}^{\infty} \log |\phi(u)| u^{-2} d u=-\pi^{2} A
$$

It follows from (3.1) that the ratio $\Lambda(t) / t$ is bounded. Hence, by (2.5) and (2.9),

$$
\begin{aligned}
\int_{-y}^{y} \log |\phi(u)| u^{-2} d u=-\frac{\pi^{2}}{y} \int_{0}^{\infty} N_{2}\left(\frac{t}{y}\right) d \Lambda(t) \\
=\frac{\pi^{2}}{y} \int_{0}^{\infty} \Lambda(t) d_{t} N_{2}\left(\frac{t}{y}\right)=-2 \int_{0}^{\infty} t^{-2} \Lambda(t) \log \left|\frac{y+t}{y-t}\right| d t \\
=O\left\{\int_{0}^{y} \frac{d t}{t} \log \left|\frac{1+t / y}{1-t / y}\right|+\int_{y}^{\infty} \frac{d t}{t} \log \left|\frac{1+y / t}{1-y / t}\right|\right\}=O(1) .
\end{aligned}
$$

Being combined with (3.2) this shows that

$$
\int_{-y}^{v} \log ^{-}|\phi(u)| u^{-2} d u=O(1)
$$

Hence the integral

$$
\int_{-\infty}^{\infty} \log ^{-}|\phi(u)| u^{-2} d u
$$

converges, whence, again by (3.2), the convergence of the integral (3.4) follows. Expression (1.2) for the constant $A$ of Theorem I is now immediately obtained.

Another, non-Tauberian, proof of Theorem 1 proceeds as follows. We have shown in the above discussion that the integral

$$
\int_{-\infty}^{\infty}|\log | \phi(u)|| u^{-2} d u
$$

converges. Hence the harmonic function

$$
F(u, v)=\log |\phi(u+i v)|-\frac{1}{\pi} \int_{-\infty}^{\infty} \frac{v \log \left|\phi\left(u^{\prime}\right)\right|}{\left(u^{\prime}-u\right)^{2}+v^{2}} d u^{\prime}
$$


exists in the upper half-plane $v>0$, and vanishes for $v=0$ (except at the zeros of $\left.\phi(w), u=\lambda_{n}{ }^{2}\right)$. It may be extended by reflection to the lower halfplane. The resulting harmonic function will be continuous everywhere, even at the zeros of $\phi(w)$. Indeed, $F(u, v)$ vanishes along the segment of the line $v=0$ through such a point, and thus cannot have a logarithmic singularity there, while the order of singularity cannot be greater than logarithmic. Thus $F(u, v)$ is the real part of an entire function.

By (1.1),

$$
\log |\phi(z)|=O(|z|) \text {. }
$$

Now,

$$
\begin{aligned}
& \int_{-\infty}^{\infty} \frac{v \log \left|\phi\left(u^{\prime}\right)\right|}{\left(u^{\prime}-u\right)^{2}+v^{2}} d u^{\prime}=\int_{-\infty}^{-2 u}+\int_{-2 u}^{2 u}+\int_{2 u}^{\infty} \\
& \quad \leqq 8 v \int_{-\infty}^{\infty}|\log | \phi\left(u^{\prime}\right)|| u^{\prime-2} d u^{\prime}+\text { const } \int_{-2 u}^{2 u} \frac{u^{\prime} v}{\left(u^{\prime}-u\right)^{2}+v^{2}} d u^{\prime} \\
& \quad=O(|z|) .
\end{aligned}
$$

Thus we must have

$$
F(u, v)=\pi A v, . v>0,
$$

for some $A$. Thus, as $v \rightarrow \infty$,

$$
\log \phi(i v)=\log |\phi(i v)|=\pi A v+\frac{1}{\pi} \int_{-\infty}^{\infty} \frac{v \log \left|\phi\left(u^{\prime}\right)\right|}{u^{\prime 2}+v^{2}} d u^{\prime}=\pi A v+o(v)
$$

which is the desired result.

Proof of Theorem II. If $\left\{z_{p}\right\}$ is the sequence of zeros of $f(z)$ we have

We set

$$
f(z)=\prod_{v=1}^{\infty}\left(1-\frac{z}{z_{p}}\right) \text {. }
$$

$$
\begin{aligned}
& \psi(w)=f\left(w^{2}\right)=\prod_{v=1}^{\infty}\left(1-\frac{w^{2}}{z_{\nu}}\right), \\
& f^{*}(z)=\prod_{\nu=1}^{\infty}\left(1-\frac{z}{\lambda_{\nu}^{2}}\right), \quad\left|z_{\nu}\right|=\lambda_{\nu}^{2}, \\
& \phi(w)=f^{*}(z)=\prod_{v=1}^{\infty}\left(1-\frac{w^{2}}{\lambda_{\nu}^{2}}\right), z=w^{2} .
\end{aligned}
$$

Then, since $\sum_{1}^{\infty} \lambda_{\nu}^{-2}<\infty$,

$$
n_{f}\left(r^{2}\right)=n_{f}^{*}\left(r^{2}\right) \sim \pi^{-1} \log M_{f^{*}}\left(r^{2}\right)=\pi^{-1} \log M_{\phi}(r)=\pi^{-1} \log \phi(i r) .
$$


Hence, by hypothesis (1.5) of Theorem II,

$$
\log \phi(i r) \sim \pi B
$$

and, by Lemma 2.1,

$$
\int_{-\infty}^{\infty} \log |\phi(u)| u^{-2} d u=-\pi^{2} B
$$

By hypothesis (1.4),

$$
-\pi^{2} B=\int_{0}^{\infty} \log |f(x)| x^{-3 / 2} d x=\int_{-\infty}^{\infty} \log |\psi(u)| u^{-2} d u .
$$

Thus we must have

$$
\int_{-\infty}^{\infty}[\log |\psi(u)|-\log |\phi(u)|] u^{-2} d u=0 .
$$

On the other hand,

$$
\log |\psi(u)|-\log |\phi(u)|=\sum_{v=1}^{\infty} \log \frac{\left|z_{\nu}-u^{2}\right|}{|| z_{\nu}\left|-u^{2}\right|}>0,
$$

unless all roots $z_{p}$ are real and positive. Thus relation (3.5) implies that all roots $z_{v}$ are positive, and Theorem II is proved.

4. In this paragraph we use the notation of $\$ 2$, but make a slightly different assumption concerning the asymptotic behavior of $\phi(i y)$.

LEMMA 4.1. If the series $\sum_{1}^{\infty} \lambda_{\nu}^{-2}$ converges, then the statements

$$
\log \phi(i y) \sim \pi A|y| \log |y| \text { as }|y| \rightarrow \infty
$$

and

$$
\int_{-y}^{y} \log |\phi(x)| x^{-2} d x \sim-\pi^{2} A \log |y|
$$

are completely equivalent.

We assume $y>0$. Using the kernels $N_{1}(\lambda), N_{2}(\lambda)$ of $\$ 2$ we may replace (4.1), (4.2) respectively by

$$
(y \log y)^{-1} \int_{0}^{\infty} N\left(\frac{t}{y}\right) d \Lambda(t) \rightarrow A, N(\lambda)=N_{1}(\lambda), N_{2}(\lambda) .
$$

We now observe that either of the statements (4.3) implies

$$
\Lambda(y)=O(y \log y) \text {. }
$$

Indeed if (4.3) is satisfied with $N=N_{1}$ or $N=N_{2}$, then 


$$
\begin{aligned}
O(1) & \geqq(y \log y)^{-1} \int_{0}^{y} N\left(\frac{t}{y}\right) d \Lambda(t) \\
& =N(1)(y \log y)^{-1} \Lambda(y)-(y \log y)^{-1} \int_{0}^{y} \Lambda(t) d_{t} N\left(\frac{t}{y}\right) \\
& >N(1) \Lambda(y)(y \log y)^{-1},
\end{aligned}
$$

since $N(\lambda)$ is positive and decreasing. Next we prove that, under the condition (4.4), (4.3) is equivalent to

$$
\frac{1}{y} \int_{0}^{\infty} N\left(\frac{t}{y}\right) d \Lambda^{*}(t) \rightarrow A, \quad N(\lambda)=N_{1}(\lambda), N_{2}(\lambda),
$$

where

$$
\Lambda^{*}(y)=\int_{0}^{y}(\log t)^{-1} d \Lambda(t)
$$

It is readily seen from (4.4) and (4.6) that $\Lambda^{*}(y)$ vanishes for sufficiently small $y$, while

$$
\Lambda^{*}(y)=O(y) \text { as } y \rightarrow \infty \text {. }
$$

Now the difference of the left-hand members of (4.5) and (4.3) is equal to

$$
\begin{aligned}
I(y) & =\frac{1}{y} \int_{0}^{\infty} N\left(\frac{t}{y}\right)\left(\frac{1}{\log t}-\frac{1}{\log y}\right) d \Lambda(t) \\
& =(y \log y)^{-1} \int_{0}^{\infty} N\left(\frac{t}{y}\right) \log \frac{y}{t} d \Lambda^{*}(t) \\
& =-(y \log y)^{-1} \int_{0}^{\infty} \Lambda^{*}(t) d_{t}\left[N\left(\frac{t}{y}\right) \log \frac{y}{t}\right] \\
& =O\left\{(\log y)^{-1} \int_{0}^{\infty} t \frac{d}{d t}\left[N(t) \log \frac{1}{t}\right] d t\right\}=O\left(\frac{1}{\log y}\right),
\end{aligned}
$$

and $\rightarrow 0$ as $y \rightarrow \infty$ or $y \rightarrow 0$. The same theorem of Wiener which was applied in the proof of Lemma 2.1 shows immediately the equivalence of the two statements (4.5); hence the two statements (4.3), and consequently (4.1) and (4.2), are also equivalent.

In order to apply Lemma 4.1 to the theory of the Riemann zeta-function we introduce 


$$
\begin{aligned}
Z(z) & =\xi\left(\frac{1}{2}+i z\right) \\
& =\frac{1}{2}\left(\frac{1}{2}+i z\right)\left(\frac{1}{2}-i z\right) \pi^{-1 / 4-i z / 2} \Gamma\left(\frac{1}{4}+\frac{i z}{2}\right) \zeta\left(\frac{1}{2}+i z\right) .
\end{aligned}
$$

It is known that $\Xi(z)$ is an entire function, is even and has all zeros in the strip $|\Im(z)|<\frac{1}{2}$. Moreover

$$
\begin{aligned}
\log \Xi(i y) & =O(y)+\log \Gamma(y / 2) \sim \frac{1}{2} y \log y, \\
\Xi(z) & =c \prod_{\nu=1}^{\infty}\left(1-\frac{z^{2}}{z_{\nu}^{2}}\right), \quad \sum_{1}^{\infty}\left|z_{\nu}\right|^{-2}<\infty, c=\Xi(0) .
\end{aligned}
$$

We set

$$
z_{\nu}=z_{\nu}^{\prime}+i z_{\nu}^{\prime}, z_{\nu}^{\prime \prime}>0,\left|z_{\nu}^{\prime \prime}\right|<\frac{1}{2} ;\left|z_{\nu}\right|=\lambda_{\nu} .
$$

Let us put

$$
\mathrm{H}(z)=c \prod_{\nu=1}^{\infty}\left(1-\frac{z^{2}}{\lambda_{\nu}^{2}}\right)
$$

We have outside the strip $|\Im(z)| \leqq 1$,

$$
\begin{aligned}
\log \left|\frac{\mathrm{H}(z)}{\Xi(z)}\right| & =-\sum_{\nu=1}^{\infty} \log \left|\frac{z_{\nu}^{2}-z^{2}}{\lambda_{\nu}{ }^{2}-z^{2}}\right|=-\sum_{\lambda_{\nu} \leqq|2 z|}-\sum_{\lambda_{\nu}>|2 z|} \\
& =\sum_{\lambda_{\nu}<|2 z|} O\left(\frac{|z|}{\lambda_{\nu}{ }^{2}}\right)+\sum_{\lambda_{\nu}>|2 z|} O\left(\frac{|z|^{2}}{\lambda_{\nu}{ }^{2}}\right)=O(|z|) .
\end{aligned}
$$

Thus, assuming $y>0$,

$$
\log \mathrm{H}(i y) \sim \frac{1}{2} y \log y,
$$

and, by Lemma 4.1,

$$
(\log y)^{-1} \int_{-y}^{y} \log \left|c^{-1} \mathrm{H}(x)\right| x^{-2} d x \rightarrow-\frac{\pi}{2} \text { as } y \rightarrow \infty .
$$

Again, on the real axis,

whence

$$
\left|1-\frac{x^{2}}{z_{\nu}^{2}}\right| \geqq\left|1-\frac{x^{2}}{\lambda_{\nu}^{2}}\right|
$$

$$
\log \left(\left|1-x^{2} / z_{\nu}^{2}\right| /\left|1-x^{2} / \lambda_{\nu}^{2}\right|\right) \geqq 0
$$

Furthermore 


$$
\begin{aligned}
0 & \leqq I_{\nu}=\int_{-\infty}^{\infty} \log \frac{\left|1-\frac{x^{2}}{z_{\nu}^{2}}\right|}{1-\frac{x^{2}}{\lambda_{\nu}^{2}} \mid} x^{-2} d x \\
& \leqq \frac{2}{\lambda_{\nu}^{2}} \int_{0}^{\infty} \log \left|\frac{1+t^{2}}{1-t^{2}}\right| t^{-2} d t=O\left(\frac{1}{\lambda_{\nu}^{2}}\right),
\end{aligned}
$$

hence we can integrate term-wise and obtain

$$
0<\int_{-\infty}^{\infty} \log \left|\frac{\Xi(x)}{\mathrm{H}(x)}\right| x^{-2} d x=\sum_{\nu=1}^{\infty} I_{\nu}<\infty .
$$

Then, by (4.8),

$$
(\log y)^{-1} \int_{-y}^{y} \log \left|c^{-1} \Xi(x)\right| x^{-2} d x \rightarrow-\frac{\pi}{2} .
$$

If we return to the zeta-function using (4.7), this gives our final result

$$
\int_{1}^{y} \frac{\log \left|\zeta\left(\frac{1}{2}+i x\right)\right|}{x^{2}} d x=o(\log y) .
$$

5. Titchmarsh has $\dagger$ discussed asymptotic properties of entire functions with real negative zeros. In this paragraph we indicate some results which overlap with those of Titchmarsh. The method used in deriving these results is closely analogous to that used in proving Lemma 2.1 ; therefore we shall give here only a brief outline of the proof leaving the details to the reader.

Let

$$
f(z)=\prod_{n=1}^{\infty}\left(1+\frac{z}{a_{v}}\right)
$$

be an entire function all of whose zeros $\left\{-a_{\nu}\right\}$ are negative. It will be assumed that

$$
0<a_{1} \leqq a_{2} \leqq \cdots, \sum_{\nu=1}^{\infty} a_{\nu}^{-1}<\infty .
$$

For simplicity we shall use the symbol $n(r)$ instead of $n_{f}(r)$ of the preceding paragraphs. The letter $x$ will designate a real positive variable which tends to infinity.

† Loc. cit. 
LEMMA 5.1. Let $\lambda, \rho, \theta$ be fixed numbers such that

$$
\lambda>0, \quad 0<\rho<1, \quad|\theta|<\pi .
$$

Then the statements

$$
\begin{aligned}
n(x) & \sim \lambda x^{\rho}, \\
\log f(x) & \sim \pi \lambda \operatorname{cosec} \pi \rho x^{\rho}, \\
\log \left|f\left(x e^{i \theta}\right)\right| & \sim \pi \lambda \operatorname{cosec} \pi \rho \cos \theta \rho x^{\rho}, \\
\int_{0}^{x} r^{-1-\pi /(2 \theta)} \log \left|f\left(r e^{i \theta}\right)\right| d r & \sim-\frac{\pi \lambda \operatorname{cosec} \pi \rho \cos \theta \rho}{\rho-\frac{\pi}{2 \theta}} x^{\rho-\pi /(2 \theta)}
\end{aligned}
$$

are all equivalent. In the last statement (iv) the right-hand member in the case $\theta=\pi /(2 \theta)$ should be replaced by its limiting value as $\rho \rightarrow \pi /(2 \theta)$.

We first observe that the convergence of the series $\sum_{1}^{\infty} a_{\nu}^{-1}$ implies

$$
n(x)=o(x) \text {. }
$$

Next let us put

$$
\omega(x)=x^{-\rho} n(x) .
$$

In view of the fact that $n(x)$ is monotone increasing it is readily seen that the statements (i), which can be written as

$$
\omega(x) \rightarrow \lambda,
$$

and

$$
\int_{0}^{x} \omega(r) d r \sim \lambda x
$$

are equivalent. $\dagger$

Our next step is to transform the left-hand members of (ii-iv) in such a way as to allow an immediate application of Wiener's Tauberian theorems. We have

$$
\begin{aligned}
x^{-\rho} \log f(x) & =x^{-\rho} \int_{0}^{\infty} \log \left(1+\frac{x}{t}\right) d n(t) \\
& =x^{-\rho} \int_{0}^{\infty} n(t) \frac{x}{t(t+x)} d t=\frac{1}{x} \int_{0}^{\infty} \omega(t) \frac{\left(\frac{t}{x}\right)^{\rho-1}}{1+\frac{t}{x}} d t,
\end{aligned}
$$

$\dagger$ This is readily proved directly or derived from a theorem of Wiener, loc. cit., Theorem XIII, pp. 34-35; it also follows from a well known theorem of Landau, Beitrage zuranalytischen Zahlentheorie, Rendiconti del Circolo Matematico di Palermo, vol. 26 (1908), pp. 169-302; p. 218. 


$$
\begin{aligned}
x^{-\rho} \log \left|f\left(x e^{i \theta}\right)\right| & =\frac{1}{2} x^{-\rho} \int_{0}^{\infty} \log \left|1+\frac{x}{t} e^{i \theta}\right|^{2} d n(t) \\
& =\frac{1}{x} \int_{0}^{\infty} \omega(t)\left(\frac{t}{x}\right)^{\rho-1} \frac{1+\frac{t}{x} \cos \theta}{1+\frac{2 t}{x} \cos \theta+\frac{t^{2}}{x^{2}} d t} \\
x^{\pi /(2 \theta)-\rho} \int_{0}^{x} r^{-1-x /(2 \theta)} \log \left|f\left(r e^{i \theta}\right)\right| d r & \int_{0}^{x} r^{-1-\pi /(2 \theta)} d r r^{\rho-1} \int_{0}^{\infty} \omega(t)\left(\frac{t}{r}\right)^{\rho-1} \frac{1+\frac{t}{r} \cos \theta}{2 t} \cos \theta+\frac{t^{2}}{r^{2}} \\
= & x^{\pi /(2 \theta)-\rho} \\
= & -\frac{1}{x} \int_{0}^{\infty} \omega(t) d t\left(\frac{x}{t}\right)^{1-\rho+\pi /(2 \theta)} \int_{x / t}^{\infty} \frac{1+\frac{2}{r} \cos \theta+\frac{1}{r^{2}} \cos \theta}{r^{-1-\pi /(2 \theta)} d r}
\end{aligned}
$$

(see (5.9) below, for $u=0, \theta \rho=\pi / 2$ ). Thus all the statements (ii-iv) are expressible in the form

$$
\frac{1}{x} \int_{0}^{\infty} \omega(t) N\left(\frac{t}{x}\right) d t \rightarrow \lambda \text { as } x \rightarrow \infty,
$$

where $N(y)$ stands, respectively, for

$$
\begin{aligned}
& N_{3}(y)=\frac{1}{\pi \operatorname{cosec} \pi \rho} \frac{y^{\rho-1}}{1+y}, \\
& N_{8}(y)=\frac{1}{\pi \operatorname{cosec} \pi \rho \cos \theta \rho} y^{\rho-1} \frac{1+y \cos \theta}{1+2 y \cos \theta+y^{2}}, \\
& N_{8}(y)=-\frac{\pi-\frac{\pi}{2 \theta}}{\pi \operatorname{cosec} \pi \rho \cos \theta \rho} y^{\rho-1-\pi /(2 \theta)} \int_{1 / y}^{\infty} \frac{1+\frac{1}{r} \cos \theta}{\frac{2}{r} \cos \theta+\frac{1}{r^{2}}} r^{-1-x /(2 \theta)} d r .
\end{aligned}
$$

A direct computation yields 


$$
\begin{gathered}
\int_{0}^{\infty} \frac{y^{i u+\rho-1}}{1+y} d y=\pi \operatorname{cosec} \pi(i u+\rho) \\
\int_{0}^{\infty} \frac{y^{i u+\rho-1}(1+y \cos \theta)}{1+2 y \cos \theta+y^{2}} d y \\
=\frac{1}{2}\left[\int_{0}^{\infty} \frac{y^{i u+\rho-1}}{1+y e^{i \theta}} d y+\int_{0}^{\infty} \frac{y^{i u+\rho-1}}{1+y e^{-i \theta}} d y\right] \\
=\int_{0}^{\infty} \frac{y^{i u+\rho-1}}{1+y} \frac{1}{2}\left[\left(e^{i \theta}\right)^{-i u-\rho}+\left(e^{-i \theta}\right)^{-i u-\rho}\right] d y \\
=\pi \operatorname{cosec} \pi(i u+\rho) \cos \theta(i u+\rho) \\
\int_{0}^{\infty} y^{i u+\rho-1-\pi /(2 \theta)} d y \int_{1 / y}^{\infty} r^{-1-\pi /(2 \theta)}-1+\frac{1}{r} \cos \theta \\
=\int_{0}^{\infty} r^{-1+\pi /(2 \theta)} \frac{1+r \cos \theta}{1+2 r \cos \theta+r^{2}} d r \int_{r}^{\infty} y^{i u+\rho-1-\pi /(2 \theta)} d y \\
=-\frac{\pi \operatorname{cosec} \pi(i u+\rho) \cos \theta(i u+\rho)}{r^{2}} d r \\
i u+\rho-\frac{\pi}{2 \theta}
\end{gathered}
$$

The last result is first derived in the case where $0<\rho<1+\pi /(2 \theta)$, but is easily extended to the general case $0<\rho<1$ by analytic continuation. It is an easy matter to verify that the kernels $N_{3}(y), N_{4}(y)$ when $|\theta|<\pi / 2$, and $N_{5}(y)$ are possessed of all the properties of the kernel $N(y)$ stated in the proof of Lemma 2.1. We set $\Lambda(t)=\int_{0}^{t} \omega(t) d t$. Since $\omega(t) \geqq 0, \Lambda(t)$ is monotone increasing. Hence Wiener's theorem used in $\$ 2$ may be applied here with the result that the statements (ii), (iii) when $|\theta|<\pi / 2$, and (iv) are equivalent, while either of (ii) or (iv) implies (iii) when $\pi / 2<|\theta|<\pi$. It should be observed that the kernel $N_{4}(y)$ is not positive when $|\theta|>\pi / 2$, while $N_{5}(y)$ is positive over the whole range $|\theta|<\pi$. The introduction of this kernel was necessitated by the lack of positiveness of $N_{4}(y)$ when $|\theta|>\pi / 2$. Another theorem of Wiener $\dagger$ will show that either of the statements (ii), (iii) when $|\theta|<\pi / 2$, and (iv) implies (5.6), hence (5.5) which is the same as (i). On the other hand it may be proved directly $\ddagger$ that (i) implies (ii), hence also (iii) and (iv). This completes the proof of Lemma 5.1.

$\dagger$ N. Wiener, loc. cit., Theorem XI", pp. 31-32.

$\ddagger$ Titchmarsh, loc. cit., Theorem I. 
VI. ON Two PROBlems of PGLya

1. Pólya $\nmid$ has set the following problem: Let the real numbers $m_{1}, m_{2}, \cdots$ have the properties $0<m_{1}<m_{2}<\cdots$ and

$$
\varliminf_{n \rightarrow \infty} \frac{n}{m_{n}}>\frac{b-a}{2 \pi}>0 .
$$

Furthermore, let $f(x)$ be continuous in the closed interval $[a, b]$. Then it will follow from

$$
\int_{a}^{b} f(x) \cos m_{n} x d x=\int_{a}^{b} f(x) \sin m_{n} x d x=0
$$

that $f(x)$ vanishes identically.

There is no restriction in supposing $b=-a=\pi$. We shall prove the following more general theorem:

ThEOREM I. Let $0<m_{1}<m_{2}<\cdots$ and let

$$
\varlimsup_{n \rightarrow \infty} \frac{n}{m_{n}}>1 \text {. }
$$

Then if $f(x)$ belongs to $L^{2}$ and

$$
\int_{-\pi}^{\pi} f(x) e^{ \pm i m_{n} x} d x=0 \quad(n=1,2,3, \cdots),
$$

$f(x)$ vanishes except over a set of measure zero.

It is very important that we have replaced $\underline{\lim }$ by $\overline{l i m}$. This yields us a much deeper theorem.

Since (1.4) is satisfied with $f(x)$ replaced by $f(x) \pm f(-x)$, it suffices to consider only the cases of $f(x)$ even or odd. We shall give the discussion of the case $f(x)$ even, under the additional assumption that $\int_{-\pi}^{\pi} f(t) d t \neq 0$. The case where this assumption is not satisfied as well as the case of $f(x)$ odd will require but slight modifications which may be left to the reader. We set

$$
\phi(u)=\int_{-\pi}^{\pi} f(t) e^{i u t} d t,
$$

where the entire function $\phi(u)$ is even and where we may assume without loss of generality that $\phi(0)=1$. We observe that, on setting $u=\sigma+i \tau$, we have

$\dagger$ Jahresbericht der Deutschen Mathematiker-Vereinigung, vol. 40 (1931), Abteilung 2, p. 81, Problem 108. 
(1.6) $|\phi(u)|=|\phi(\sigma+i \tau)| \leqq\left\{\int_{-\pi}^{\pi}|f(t)|^{2} d t\right\}^{1 / 2} e^{\pi|\tau|}=O\left(e^{\pi|\tau|}\right)$.

On the other hand by the theory of Fourier transforms we know that $\phi(\sigma) \subset L^{2}$ over $(-\infty, \infty)$ and that the Fourier transform of $\phi(\sigma)$ vanishes outside $(-\pi, \pi)$. Hence by Theorem II of our Note I, $\dagger$

$$
\int_{-\infty}^{\infty} \frac{|\log | \phi(\sigma)||}{1+\sigma^{2}} d \sigma<\infty .
$$

By the change of variable $u^{2}=z$ we obtain a function $\psi(z)=\phi(u)$ which satisfies the conditions of Theorem I of our preceding Note V. It follows at once that the limit

$$
\lim _{r \rightarrow \infty} \frac{n_{\phi}(r)}{r}=A
$$

exists. Let $\left\{u_{\nu}\right\}$ be the sequence of zeros of $\phi(u)$. It is clear that $\left\{ \pm m_{\nu}\right\}$ is a subsequence of $\left\{u_{\nu}\right\}$. Hence, by (1.3),

$$
2<\varlimsup_{n \rightarrow \infty} \frac{2 n}{m_{n}} \leqq \lim _{r \rightarrow \infty} \frac{n_{\phi}(r)}{r}=A .
$$

However, by Jensen's theorem, in view of.(1.6),

$$
\begin{aligned}
\frac{1}{r} \int_{0}^{r} \frac{n_{\phi}(t)}{t} d t & =\frac{1}{2 \pi r} \int_{0}^{2 \pi} \log \left|\phi\left(r e^{i \theta}\right)\right| d \theta \\
& \leqq \frac{1}{2 \pi r} \int_{0}^{2 \pi} \pi r|\sin \theta| d \theta+O\left(\frac{1}{r}\right)=2+o\left(\frac{1}{r}\right) .
\end{aligned}
$$

Hence

$$
A=\lim _{r \rightarrow \infty} \frac{1}{r} \int_{0}^{r} \frac{n_{\phi}(t)}{t} d t \leqq 2 .
$$

The resulting contradiction shows that $f(x)$ must vanish except for a set of measure zero.

2. Pólyał has also set the following problem: Let $f(z)$ be an entire function which is bounded for the integral arguments $z=0, \pm 1, \pm 2, \cdots, \pm n, \cdots$. Let

$$
M_{f}(r)=o(r) .
$$

Then $f(z)$ reduces to a constant.

† The present volume of these Transactions, p. 349 .

$\ddagger$ Jahresbericht der Deutschen Mathematiker-Vereinigung, vol. 40 (1931), Abteilung 2, p. 80, Problem 105. 
It is clearly sufficient to prove the theorem for an even $f(z)$, for if $f(z)$ is odd, we need only consider $f(z) / z$, which will be even, and will hence reduce to a constant which can only be zero. The general function may then be treated by reducing it to the sum of an odd and an even part.

If $f(z)$ is even,

$$
g(z)=[f(z)-f(0)] z^{-2}
$$

will be entire. Thus

$$
\sum_{n=-\infty}^{\infty}|g(n)|<\infty
$$

Let us form

$$
G(z)=\sum_{n=-\infty}^{\infty} g(n) \frac{\sin \pi(n-z)}{\pi(n-z)}
$$

Clearly

$$
G(x+i y)=O\left(y^{-1} e^{x|y|}\right) .
$$

Let us now form the entire function

$$
H(z)=[g(z)-G(z)] \operatorname{cosec} \pi z .
$$

For all values of $z$ and all integral values of $n$ we shall have

$$
\begin{aligned}
H\left[\left(n+\frac{1}{2}\right)+i y\right]= & O\left\{\exp \left(\epsilon\left[\left(n+\frac{1}{2}\right)^{2}+y^{2}\right]^{1 / 2}-\pi|y|\right)\right\} \\
& +O(1 /|y|) \\
= & e^{\epsilon|n+1 / 2|} O\left(e^{(\epsilon-x)|y|}\right)+O(1 /|y|)
\end{aligned}
$$

uniformly in $n$. We have here employed (2.1) and (2.5). Hence

$$
\int_{-\infty}^{\infty}\left|H\left(n+\frac{1}{2}+i y\right)\right|^{2} d y=O\left(e^{2 \epsilon|n|}\right)
$$

for all $\epsilon$.

Let us put

$$
x_{1}=\left[x+\frac{1}{2}\right], x_{2}=\left[x-\frac{1}{2}\right] .
$$

Then, by Cauchy's theorem,

$$
\begin{aligned}
H(x+i y)=(2 \pi i)^{-1} \int_{-\infty}^{\infty} & \frac{H\left(x_{1}+\frac{1}{2}+i y_{1}\right)}{x_{1}+\frac{1}{2}+i y_{1}-x-i y} d y_{1} \\
& -(2 \pi i)^{-1} \int_{-\infty}^{\infty} \frac{H\left(x_{2}-\frac{1}{2}+i y_{1}\right)}{x_{2}-\frac{1}{2}+i y_{1}-x-i y} d y_{1} .
\end{aligned}
$$

Hence 


$$
\begin{aligned}
& \int_{-\infty}^{\infty} H(x+i y) e^{i u y} d y \\
= & (2 \pi i)^{-1} \int_{-\infty}^{\infty} H\left(x_{1}+\frac{1}{2}+i y_{1}\right) e^{i u y_{1} d y_{1}} \int_{-\infty}^{\infty} \frac{e^{i u y} d y}{x_{1}+\frac{1}{2}-x-i y} \\
& -(2 \pi i)^{-1} \int_{-\infty}^{\infty} H\left(x_{2}-\frac{1}{2}+i y_{1}\right) e^{i u y_{1}} d y_{1} \int_{-\infty}^{\infty} \frac{e^{i u y}}{x_{2}-\frac{1}{2}-x-i y},
\end{aligned}
$$

and, by the Plancherel theorem and the Schwarz unequality,

$$
\begin{aligned}
\int_{-\infty}^{\infty}|H(x+i y)|^{2} d y \leqq & \text { const }\left\{\int_{-\infty}^{\infty}\left|H\left(x_{1}+\frac{1}{2}+i y\right)\right|^{2} d y\right. \\
& \left.+\int_{-\infty}^{\infty}\left|H\left(x_{2}-\frac{1}{2}+i y\right)\right|^{2} d y\right\} .
\end{aligned}
$$

Thus, by (2.8),

$$
\int_{-\infty}^{\infty}|H(x+i y)|^{2} d y=O\left(e^{2 e|x|}\right) .
$$

By an application of Cauchy's theorem,

$$
\int_{-\infty}^{\infty} H(x+i y) e^{i u y} d y=e^{-u x} \int_{-\infty}^{\infty} H(i y) e^{i u y} d y \equiv e^{-u x} \phi(y) .
$$

Thus by the Plancherel theorem,

$$
\int_{-\infty}^{\infty}|\phi(u)|^{2} e^{-2 u x} d u=O\left(e^{2 e|x|}\right) .
$$

This is however only possible if $\phi(u)$ vanishes almost everywhere for $|u|>\epsilon$. Since $\epsilon$ is arbitrarily small, $\phi(u)$ must be equivalent to zero. Thus $H(z)$ vanishes, and $g(z)=G(z)$. On the other hand,

$$
M_{G}(r) \sim \pi r
$$

unless every $g(n)$ is zero. This yields

$$
G(z)=g(z)=0, f(z)=f(0),
$$

which is the desired result.

\section{ON THE VOLTERRA EQUATION}

1. A theorem of Mercert asserts that if $0<\alpha<1$, and if

$\dagger$ J. Mercer, On the limits of real variants, Proceedings of the London Mathematical Society, (2), vol. 5 (1907), pp. 206-224. 


$$
\alpha s_{n}+(1-\alpha) \frac{1}{n} \sum_{1}^{n} s_{p} \rightarrow s,
$$

then

$$
s_{n} \rightarrow s .
$$

This theorem possesses generalizations of a non-trivial nature. The continuous analogue asserts that if $0<\alpha<1$ and if

$$
\alpha s(x)+\frac{1-\alpha}{x} \int_{1}^{x} s(y) d y \rightarrow s \text { as } x \rightarrow \infty,
$$

then

$$
s(x) \rightarrow s .
$$

By a change of independent variable, this asserts that if

$$
\alpha S(\xi)+(1-\alpha) \int_{0}^{\xi} e^{\eta-\xi} S(\eta) d \eta \rightarrow s,
$$

then

$$
S(\xi) \rightarrow s .
$$

This statement is a particular case of the following theorem:

THEOREM I. Let $F(x)$ be measurable and bounded over every finite range $(0, A)$. Let $K(x) \subset L$ over $(0, \infty)$, that is,

$$
\int_{0}^{\infty}|K(\xi)| d \xi<\infty .
$$

Let

$$
F(x)+\int_{0}^{x} K(x-\xi) F(\xi) d \xi \rightarrow s \text { as } x \rightarrow \infty
$$

Then if

$$
\int_{0}^{\infty} K(\xi) e^{-w \xi} d \xi \neq-1, \Re(w) \geqq 0,
$$

we shall have

$$
F(x) \rightarrow s\left[1+\int_{0}^{\infty} K(\xi) d \xi\right] .
$$

Conversely, let $K(x) \subset L, \int_{0}^{\infty} K(\xi) d \xi \not-1$, and let (1.8) imply (1.10) for every $F(x)$ satisfying our conditions. Then (1.9) must be true. 
2. The second part of this theorem may be proved by reductio ad absurdum by putting

where

$$
F(x)=e^{w_{0} x}
$$

Then

$$
\int_{0}^{\infty} K(\xi) e^{-w_{0} \xi} d \xi=-1, \Re\left(w_{0}\right) \geqq 0, w_{0} \neq 0 \text {. }
$$

$$
\begin{aligned}
\left|F(x)+\int_{0}^{x} K(x-\xi) F(\xi) d \xi\right| & =\left|e^{w_{0} x} \int_{x}^{\infty} K(\xi) e^{-w_{0} \xi} d \xi\right| \\
& \leqq \int_{x}^{\infty}|K(\xi)| d \xi \rightarrow 0
\end{aligned}
$$

As (1.10) is obviously false, the second part of the theorem is proved.

3. The first part of Theorem I will appear as a corollary to a theorem concerning the Volterra integral equation of the closed cycle.

We shall use the symbol

$$
A \star B(x)
$$

to indicate the "Faltung" of the two functions $A(x), B(x)$,

$$
A \star B(x)=\int_{0}^{x} A(\xi) B(x-\xi) d \xi=\int_{0}^{x} A(x-\xi) B(\xi) d \xi=B \star A(x) .
$$

It is well known that the (bounded and measurable) solution of the Volterra integral equation

$$
G(x)=F(x)+K \star F(x)
$$

is uniquely determined and given by

$$
F(x)=G(x)+Q \star G(x)
$$

where the resolvent kernel $Q(x)$ itself is determined from

$$
Q(x)+K(x)=-K \star Q(x)=-Q \star K(x),
$$

or else, by

$$
Q(x)=\sum_{n=1}^{\infty}(-1)^{n} K^{n}(x), K^{n}(x)=K \star K \star \cdots \star K(x) .
$$

We observe that the solution of (3.3) is easily obtained by using Laplace transforms. $\uparrow$ Let us designate by

† See, e.g., S. Bochner, Vorlesungen über Fouriersche Integrale, Leipzig, 1932, chapter VII. Other references are also found there. 


$$
\begin{aligned}
& k(w)=\int_{0}^{\infty} K(\xi) e^{-w \xi} d \xi, \\
& q(w)=\int_{0}^{\infty} Q(\xi) e^{-w \xi} d \xi,
\end{aligned}
$$

the Laplace transforms of $K(x), Q(x)$. Equation (3.4) reduces then to

$$
q(w)=\frac{-k(w)}{1+k(w)},
$$

and $Q(x)$ will be found by inversion of a Laplace integral.

The theorem in question may now be stated as follows:

THEOREM II. A necessary and sufficient condition that $Q(x) \subset L$ over $(0, \infty)$, that is,

$$
\int_{0}^{\infty}|Q(\xi)| d \xi<\infty
$$

is that

$$
k(w)=\int_{0}^{\infty} K(\xi) e^{-w \xi} d \xi \neq-1, \quad \Re(w) \geqq 0 .
$$

If this theorem holds true, the first part of Theorem I is immediately derived. Indeed, under the assumptions made we have

$$
F(x)=G(x)+\int_{0}^{x} G(x-\xi) Q(\xi) d \xi .
$$

Here $G(\xi)$ is bounded over every finite range and $\rightarrow s$ as $\xi \rightarrow \infty$. Hence $G(\xi)$ is bounded over the whole range $(0, \infty)$. Since $Q(\xi)$ is integrable over $(0, \infty)$ we may pass to limit as $x \rightarrow \infty$ under the integral sign, with the result

$$
F(x) \rightarrow s+s \int_{0}^{\infty} Q(\xi) d \xi=s[1+q(0)]=s[1+k(0)]^{-1}
$$

which is precisely the desired formula (1.10).

4. To prove the necessity of (3.9) we observe that if (3.8) holds then $q(w)$ as well as $k(w)$ are analytic in the half-plane $\Re(w)>0$ and continuous up to and including the boundary $\Re(w)=0$. This implies that the denominator in the right-hand member of (3.7) does not vanish for $\Re(w) \geqq 0$, so that (3.9) holds.

The proof that (3.9) is sufficient is more difficult. We introduce the auxiliary functions 


$$
\phi_{\Lambda}(u)=\left\{\begin{array}{l}
1,|u|<A, \\
2-|u| / A, A \leqq|u| \leqq 2 A, \\
0,|u|>2 A,
\end{array}\right.
$$

and put

$$
\begin{aligned}
q^{*}(w) & \equiv \frac{-k(w)}{1+k(w)} \\
q^{*}(i u) & =q_{1}(u)+q_{2}(u) \\
q_{1}(u) & =\phi_{\Lambda}(u) q^{*}(i u), q_{2}(u)=\left[1-\phi_{A}(u)\right] q^{*}(u)
\end{aligned}
$$

We wish to prove that if $A$ is sufficiently large, $q_{1}(u)$ and $q_{2}(u)$ are both Fourier transforms of functions of $L$.

To begin with,

$$
q_{1}(u)=\left\{\begin{array}{l}
\frac{-\phi_{A}(u) k(i u)}{\phi_{2 A}(u)+\phi_{2 A}(u) k(i u)} \text { when }|u|<2 A, \\
0 \text { when }|u| \geqq 2 A .
\end{array}\right.
$$

Thus $q_{1}(u)$ is the quotient of two functions, each the Fourier transform of a function of $L$, each vanishing outside a finite range, and such that the denominator function only vanishes in points interior to the region of vanishing of the numerator function. We may then appeal to a theory due to Wienert to show that $q_{1}(u)$ is the Fourier transform of a function of $L$.

We have

$$
q_{2}(u)=\left[1-\phi_{A}(u)\right] \frac{-k(i u)\left[1-\phi_{A / 2}(u)\right]}{1+k(i u)\left[1-\phi_{A / 2}(u)\right]} .
$$

It is easy to show that this is the Fourier transform of a function of $L$ when the same is true of

$$
\begin{aligned}
& -k(i u)\left[1-\phi_{A / 2}(u)\right]\left\{1+k(i u)\left[1-\phi_{A / 2}(u)\right]\right\}^{-1} \\
& \quad=\sum_{n=1}^{\infty}(-1)^{n}\left\{k(i u)\left[1-\phi_{A / 2}(u)\right]\right\}^{n} .
\end{aligned}
$$

Now

$$
\left\{k(i u)\left[1-\phi_{A / 2}(u)\right]\right\}^{n}
$$

is the Fourier transform of a function $h_{n}(x)$ for which

$\dagger$ N. Wiener, The Fourier Integral and Certain of its A pplications, Cambridge, 1933; Lemmas 67, $6_{10}, \sigma_{18}$ 


$$
\begin{aligned}
\int_{-\infty}^{\infty}\left|h_{n}(\xi)\right| d \xi & \leqq\left[\int_{-\infty}^{\infty}\left|h_{1}(\xi)\right| d \xi\right]^{n} \\
= & {\left[\int_{-\infty}^{\infty} d \xi\left|K(\xi)-\frac{1}{\pi A} \int_{0}^{\infty} K(\eta) \frac{\cos \frac{A}{2}(\xi-\eta)-\cos A(\xi-\eta)}{(\xi-\eta)^{2}} d \eta\right|\right]^{n} . }
\end{aligned}
$$

An argument of the familiar Fejér type will show that we may choose $A$ so large that the integral in brackets is less than any given number $\lambda, 0<\lambda<1$. It will follow at once that $q_{2}(u)$ is the Fourier transform of a function $F_{2}(x)$ for which

$$
\int_{-\infty}^{\infty}\left|F_{2}(\xi)\right| d \xi \leqq \frac{\lambda}{1-\lambda} .
$$

Combining this with the similar result for $q_{1}(u)$, we see that we may write

$$
q^{*}(i u)=\frac{-k(i u)}{1+k(i u)}=\int_{-\infty}^{\infty} F(\xi) e^{-i u \xi} d \xi, F(\xi) \subset L .
$$

We may rewrite (4.5) as

$$
\int_{-\infty}^{0} F(\xi) e^{-i u \xi} d \xi=-\int_{0}^{\infty} F(\xi) e^{-i u \xi} d \xi+q^{*}(i u) .
$$

Now, it is readily seen that $k(w) \rightarrow 0$ as $|w| \rightarrow \infty$, uniformly in the halfplane $\Re(w) \geqq 0$. Since, by hypothesis, $1+k(w) \neq 0$ for $\Re(w) \geqq 0$, there exists a positive constant $c$ such that

$$
|1+k(w)| \geqq c>0 .
$$

Thust

$$
-\int_{0}^{\infty} F(\xi) e^{-w \xi} d \xi+q^{*}(w)
$$

is a function of $w$ analytic and bounded in the right half-plane, and continuous up to and including the imaginary axis. Similarly,

$$
\int_{-\infty}^{0} F(\xi) e^{-\omega \xi} d \xi
$$

is a function of $w$ analytic and bounded in the left half-plane, and continuous up to and including the imaginary axis. Furthermore, the two functions are identical on the imaginary axis. By the classical argument of RiemannPainleve it readily results that they are parts of the same analytic function, which is thus entire and bounded. It hence reduces to a constant, and since 


$$
\int_{-\infty}^{0} F(\xi) e^{-w \xi} d \xi \rightarrow 0 \text { as } w \rightarrow-\infty,
$$

this constant can only be 0 . Thus

$$
q^{*}(w)=\frac{-k(w)}{1+k(w)}=\int_{0}^{\infty} F(\xi) e^{-w \xi} d \xi .
$$

On the other hand, it follows readily from (3.4) that there exists a $w_{0}>0$ such that

$$
\frac{-k(w)}{1+k(w)}=\int_{0}^{\infty} Q(\xi) e^{-w \xi} d \xi, \Re(w)>w_{0}
$$

and

$$
\int_{0}^{\infty}|Q(\xi)| e^{-w_{0} \xi} d \xi<\infty .
$$

By the uniqueness theorem for Laplace transforms we conclude that $F(x) e^{-w x}$ and $Q(x) e^{-w x}$ coincide almost everywhere, whence $Q(x) \subset L$.

5. In this proof, we have used the theorem $\dagger$ of Wiener that if a function has an absolutely convergent Fourier series and does not vanish, its reciprocal has an absolutely convergent Fourier series. P. Lévy $\ddagger$ has pointed out that the same methods suffice for the following theorem: if a function $f(x)$ has an absolutely convergent Fourier series, and $\Phi(u)$ is analytic over the range of values of $f(x)$, then $\Phi[f(x)]$ has an absolutely convergent Fourier series. By methods not essentially different from those of this paper, we may extend this theorem as follows: if $f(x)$ is the Fourier transform of a function of $L$, and $\Phi(u)$ is analytic over the range of values of $f(x)$, including 0 , then

$$
\Phi[f(x)]
$$

is the Fourier transform of a function of $L$.

$\dagger$ Loc. cit., Lemma $\sigma_{16}$.

‡ P. Lévy, Sur la convergence absolue des séries de Fourier, Paris Comptes Rendus, vol. 196 (1933), p. 463.

Massachusetrs Institute of Technology,

Cambrimge, Mass. 